\title{
ADDIE: ADvanced DIffraction Environment - A Software Environment for Analyzing Neutron Diffraction Data
}

\author{
M. T. McDonnell ${ }^{1}$, D. P. Olds ${ }^{1}$, K. L. Page ${ }^{1}$, J. C. Neufeind ${ }^{1}$, M. G. Tucker ${ }^{1}$, J. C. Bilheux ${ }^{1}$, W. Zhou ${ }^{1}$, and P. \\ F. Peterson ${ }^{1}$ \\ ${ }^{1}$ Spallation Neutron Source, Oak Ridge National Laboratory, Oak Ridge, TN 37831, USA
}

\begin{abstract}
ADDIE is a software environment that aims to provide an intuitive graphical user interface for executing, managing, and visualizing total scattering neutron powder diffraction data. ADDIE is the current data reduction software being developed and used at the Nanoscale-Ordered Materials Diffractometer $\left(\right.$ NOMAD $^{[1]}$, a time-of-flight powder diffractometer at the Spallation Neutron Source (SNS) at Oak Ridge National Laboratory. In ADDIE, the user is provided with a workflow that begins with selection of standard, normalization, and background datasets for sample data corrections. Options are available to perform inelastic (Placzek), absorption, and multiple scattering data corrections. After initial data reduction of individual runs, the user can analyze datasets and perform summing of selected runs to provide datasets of better statistical significance. From the summed spectrum, the user can visualize the by-bank Bragg peak data to determine the average structure of materials while simultaneously visualizing the $S(Q)$ and $G(r)$ data. Finally, the user can output the optimized spectra to different file formats that feed into multiple data modelling programs.
\end{abstract}

One of the major goals of ADDIE is to deliver an accessible, simultaneous view of Bragg data, the total scattering structure factor $\mathrm{S}(\mathrm{Q})$, and the pair distribution function $\mathrm{G}(\mathrm{r})$ for data analysis. Another major goal is ease of optimization of the Fourier transform from $S(Q)$ to $G(r)$ via pre-defined filter functions and adjustable limits on $\mathrm{Q}_{\min }$ and $\mathrm{Q}_{\max }$. A Python interface is integrated into the visualization window with the Mantid framework $^{[2]}$ to provide the ability for user-defined extensions and manipulations of the datasets. The current development of ADDIE is directed towards the ability to access metadata of datasets from multiple databases at the SNS; filtering neutron events by certain sample conditions to aid in in-situ experiments; provide input files for other data reduction software for benchmarking and comparison; and increase the portfolio of output formats to support different data modelling software.

Overall, ADDIE aims to deliver an optimal user experience, increase the connectivity between data reduction and modelling software, and to optimize the data analysis process in neutron diffraction experiments.

[1] J. Neuefeind, M. Feygenson, J. Carruth, R. Hoffman, and K. K. Chipley. The Nanoscale Ordered MAterials Diffractometer NOMAD at the Spallation Neutron Source SNS. Nuclear Instruments and Methods in Physics Research Section B: Beam Interactions with Materials and Atoms. 287:68-75, 2012.

[2] O. Arnolda, b, J.C. Bilheuxc, J.M. Borregueroc, A. Butsa, S.I. Campbellc, L. Chapona, d, M. Doucetc, N. Drapera, b, R. Ferraz Leald, M.A Gigga, b, V.E. Lynchc, A. Markvardsena, D.J. Mikkelsone, c, R.L. Mikkelsone, c, R. Millerf, K. Palmena, P. Parkera, G. Passosa, T.G. Perringa, P.F. Petersonc, S. Renc, M.A. Reuterc, A.T. Savicic, , , J.W. Taylora, R.J. Taylorc, g, R. Tolchenova, b, W. Zhouc, J. Zikovskyc.. Mantid-Data analysis and visualization package for neutron scattering and $\mu$ SR experiments. Equipment. 764:156-166, 2014. 